EPJ Web of Conferences 70, 00030 (2014)

DOI: $10.1051 /$ epjconf/ 20147000030

C) Owned by the authors, published by EDP Sciences, 2014

\title{
Hard And Soft QCD Physics In ATLAS
}

\author{
Stefanie Adomeit ${ }^{1}$,a On behalf of the ATLAS Collaboration \\ ${ }^{11}$ LMU Munich, Faculty of Physics, Schellingstrasse 4, 80799 Munich, Germany
}

\begin{abstract}
Hard and soft QCD results using proton-proton collisions recorded with the ATLAS detector at the LHC are reported. Charged-particle distributions and forwardbackward correlations have been studied in low-luminosity minimum bias data taken at centre-of-mass energies of $\sqrt{s}=0.9,2.36$ and $7 \mathrm{TeV}$. Recent measurements on underlying event characteristics using charged-particle jets are also presented. The results are tested against various phenomenological soft QCD models implemented in Monte-Carlo generators. A summary of hard QCD measurements involving high transverse momentum jets is also given. Inclusive jet and dijet cross-sections have been measured at a centre-of-mass energy of $7 \mathrm{TeV}$ and are compared to expectations based on NLO pQCD calculations corrected for non-perturbative effects as well as to NLO Monte Carlo predictions. Recent studies exploiting jet substructure techniques to identify hadronic decays of boosted massive particles are reported.
\end{abstract}

\section{Introduction}

The large centre-of-mass energy proton-proton collisions at the LHC allow to study QCD processes in a kinematic regime that has never been reached before at a hadron collider. Measurements involving high- $\mathrm{p}_{\mathrm{T}}$ jets provide an important tool to test perturbative QCD (pQCD) models implemented in Monte-Carlo (MC) generators as well as NLO calculations. While the main interest at hadron colliders lies in measurements at high momentum transfer, all collider observables are affected by soft QCD processes which form an irreducible background to hard scattering events via the presence of multiple-partonic interactions (MPI). Due to their non-perturbative nature soft QCD processes require phenomenological models that need to be tuned to data. The ATLAS experiment [1] has published a wide range of measurements involving soft and hard QCD physics. These include jet production measurements giving insight into $\mathrm{PQCD}$ in the new, extended phase space region provided by the LHC as well as recent studies exploiting the use of the internal structure of jets to indentify heavily boosted object decays in searches for new physics. Measurements of observables sensitive to soft QCD processes have been carried out using ATLAS data allowing to obtain an improved understanding of soft particle production at LHC centre-of-mass energies.

\footnotetext{
ae-mail: stefanie.adomeit@physik.uni-muenchen.de
} 


\section{Soft QCD Physics in ATLAS}

\subsection{Minimum Bias Measurements}

Minimum bias (MB) categorises a class of events selected based on a minimal set of requirements ensuring that an inelastic interaction has occurred. Typically, these events are dominated by soft interactions at low momentum transfer with contributions from both diffractive and non-diffractive processes. Observables sensitive to the properties of MB events have been studied by ATLAS in low luminosity data recorded at $\sqrt{s}=0.9,2.36$ and $7 \mathrm{TeV}$ in 2009 and the beginning of 2010 [2]. Events were collected using a single-arm minimum bias scintillator trigger requiring one hit over threshold on either side of the detector.

The kinematics of charged particles have been studied using tracks reconstructed in the Inner Detector (ID). The data are corrected back to particle level using minimal model dependent corrections accounting for event selection and track reconstruction efficiencies. While it is not possible to experimentally disentangle diffractive and non-diffractive effects the measurements have been performed in three different phase space regions with a varying contribution of diffraction. Each region in phase space is defined by the minimum number of charged particles per event and minimum transverse particle momentum $\mathrm{p}_{\mathrm{T}}$. Fig. 1 shows the average charged particle multiplicity per unit of pseudorapidity in the central region $(\eta=0)$ as a function of centre-of-mass energy $\sqrt{s}$. For all three phase space regions the measured data points are compared to predictions of MC models tuned to a wide range of measurements. The AMBT1 [2] tune shows good agreement in the diffraction suppressed phase space regions with $\mathrm{p}_{\mathrm{T}}=500 \mathrm{GeV}$ whereas the pre-LHC tunes tend to show lower particle multiplicities compared to data. However, in the most inclusive phase space region $\left(\mathrm{p}_{\mathrm{T}}>100 \mathrm{GeV}, \mathrm{n}_{\mathrm{ch}} \leq 20\right)$ all MC models under study understimate the amount of activity.

\subsection{Correlation Measurements}

Correlations between charged-particle multiplicities in forward and backward pseudorapidity bins have been studied using ATLAS MB data recorded at $\sqrt{s}=0.9$ and $7 \mathrm{TeV}$ [3]. While jets originating from hard processes produce high particle multiplicites within one pseudorapidity bin, quickly diminishing as the pseudorapidity distance increases, soft processes by contrast tend to give rise to lower particle multiplicities over a wider pseudorapidity range. The forward-backward (FB) multiplicity correlation $\rho_{\mathrm{fb}}^{\mathrm{n}}$ between two particle multiplicities is defined as

$$
\rho_{f b}^{n}=\frac{\left\langle\left(n_{f}-\left\langle n_{f}\right\rangle\right)\left(n_{b}-\left\langle n_{b}\right\rangle\right)\right\rangle}{\sqrt{\left\langle\left(n_{f}-\left\langle n_{f}\right\rangle\right)^{2}\right\rangle\left\langle\left(n_{b}-\left\langle n_{b}\right\rangle\right)^{2}\right\rangle}}
$$

where $\mathrm{n}_{\mathrm{f}}$ and $\mathrm{n}_{\mathrm{b}}$ are the particle multiplicities in two chosen forward and backward intervals respectively and \langle\rangle denotes the mean over the events in the sample. Fig. 2 shows the correlations between symmetrically opposite $\eta$ intervals found in the $7 \mathrm{TeV}$ data, compared to the predictions of various MC tunes. While the general shape of the data distribution is reasonably well described by the MC tunes, the strength of the correlation is found to differ by up to $15 \%$ for some of the tunes. The correlations are described most consistently by AMBT2B [4] which was tuned to different variables using the ATLAS MB data recorded at $\sqrt{s}=0.9$ and $7 \mathrm{TeV}$. Also shown in Fig. 2 is the ratio of the FB correlations at $900 \mathrm{GeV}$ and $7 \mathrm{TeV}$ for both data and different $\mathrm{MC}$ tunes. The multiplicity correlations are found to be lower at $900 \mathrm{GeV}$ with the relative difference being greater at larger pseudorapdity separations indicating significantly higher long-range correlations with increasing centre-of-mass energy. This trend is reproduced in most of the MC predictions.

Further correlation measurements using MB data have been carried out by ATLAS based on the azimuthal ordering of charged hadrons [5] as well as two-particle angular correlations [6]. 


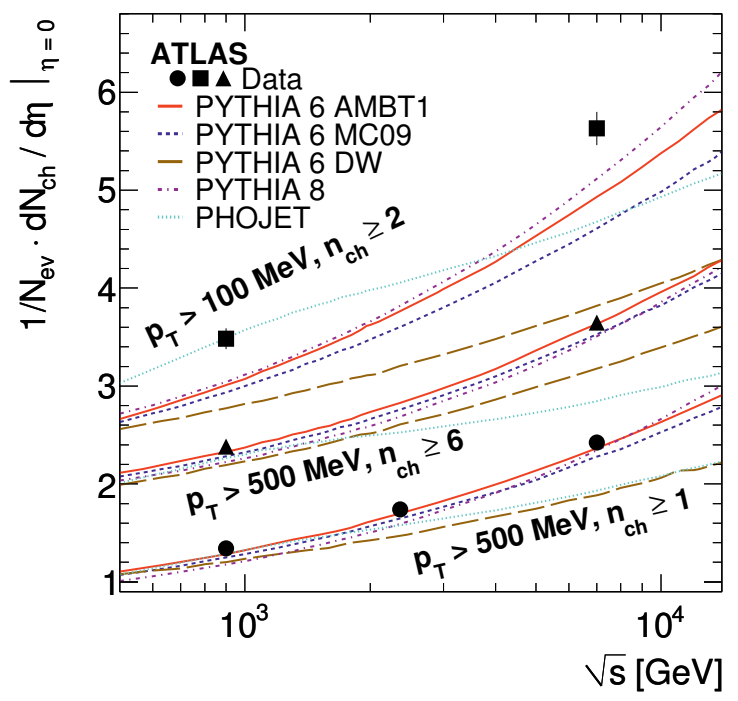

Figure 1. The average charged-particle multiplicity per unit of rapidity for $\eta=0$ as a function of the centreof-mass energy. The results with at least 2 charged particles within the kinematic range $\mathrm{p}_{\mathrm{T}}>100 \mathrm{MeV}$ and $|\eta|<2.5$ are shown alongside the results with at least 1 and 6 charged particles respectively within the kinematic range $\mathrm{p}_{\mathrm{T}}>500 \mathrm{MeV}$ and $|\eta|<2.5$ at $0.9,2.36$ and $7 \mathrm{TeV}$. The data are compared to various particle level MC predictions. The vertical error bars on the data represent the total uncertainty [2].
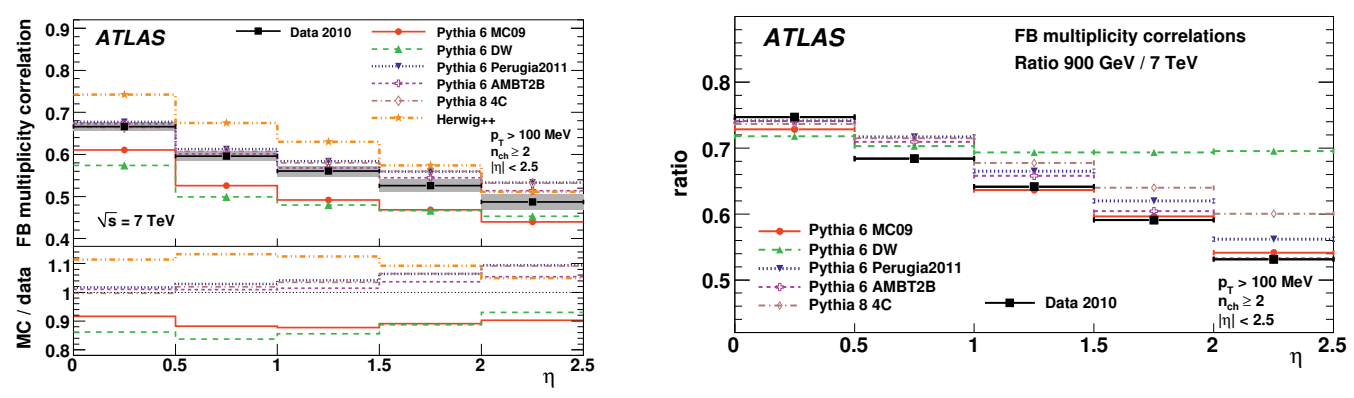

Figure 2. Forward-backward multiplicity correlation in symmetrically opposite $\eta$ intervals for events with at least two charged particles with $\mathrm{p}_{\mathrm{T}}>100 \mathrm{MeV}$ and $|\eta|<2.5$ The correlations measured in the data at $7 \mathrm{TeV}$ (left) as well as the ratio of the $900 \mathrm{GeV}$ results to the results at $7 \mathrm{TeV}$ (right) are compared to MC predictions [3].

\subsection{Underlying Event Measurements}

The underlying event (UE) is defined to be any soft activity accompanying a high $\mathrm{p}_{\mathrm{T}}$ interaction of interest thus forming an irreducible background in hard scattering events. Distributions sensitive to UE activity are typically studied in the region perpendicular to the axis defined by the hard scatter. This so-called transverse region is defined via the azimuthal angular difference $60^{\circ}<|\Delta \phi|<120^{\circ}$ relative to the direction of the hard scatter products approximated by the leading $\mathrm{p}_{\mathrm{T}}$ object. 

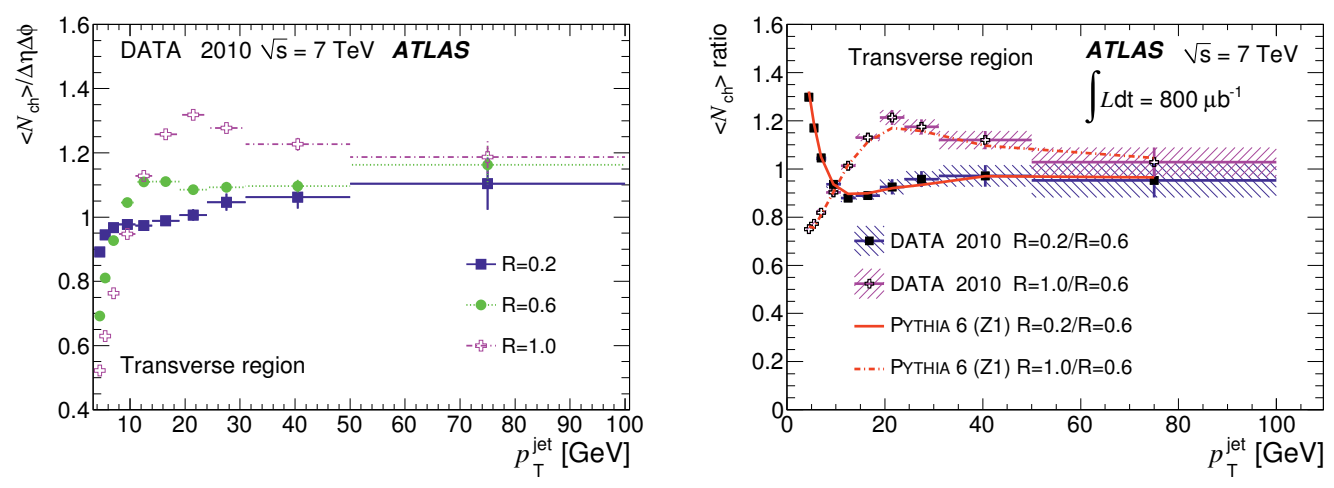

Figure 3. The mean value of the number of charged particles in the transverse region as a function of $p_{T}^{\text {jet }}$ for anti- $\mathrm{k}_{\mathrm{t}}$ jets with distance parameteres $\mathrm{R}=0.2,0.61 .0$ (left). Also shown is the ratio of the mean value obtained for distance parameters $R=0.2$ and $R=1.0$ to the results obtained for $R=0.6$ (right). The shaded bands show the total uncertainty. The lines show the predictions of Pythia 6 with the Z1 [10] tune [11].

Previous UE measurements carried out by ATLAS have used ID tracks associated to charged particles [7] as well as energy deposits in the calorimeter associated to both neutral and charged particles [8]. These results have recently been extended by UE studies based on charged-particle jets, reconstructed by applying the anti- $\mathrm{k}_{\mathrm{t}}$ algorithm [9] to ID tracks with distance parameters $\mathrm{R}$ varying between 0.2 and 1.0 [11]. Distributions of the charged particle multiplicity, the scalar sum of the transverse momenta of charged particles and the average charged-particle $\mathrm{p}_{\mathrm{T}}$ have been studied as a function of jet $\mathrm{p}_{\mathrm{T}}$ in regions transverse and opposite to the leading jet. In addition, the dependence of these distributions on the jet algorithm distance parameter $\mathrm{R}$ was investigated. Fig. 3 shows the charged-particle multiplicity in the transverse region as a function of jet $\mathrm{p}_{\mathrm{T}}$ for three representative values of $R$ as well as the ratio of the results obtained with different $R$ parameters. As the radius parameter $\mathrm{R}$ increases the jet transverse momentum $\mathrm{p}_{\mathrm{T}}$ is typically shifted towards higher values. Due to the collection of additional UE particles that contribute to the amount of bin migration any variation of the UE distributions with R additionally allows to test the ability of MC generators to model UE fluctuations and their spatial correlations. Reasonable agreement has been found between data and PYTHIA 6 [12] predictions based on tunes that have been determined using LHC data in discribing the mean UE event activity as well as in modelling coherent fluctuations.

\section{Hard QCD Physics in ATLAS}

\subsection{Jet Cross Section Measurements}

Jet cross-sections serve as an important tool for providing information on the internal structure of the proton as well as for testing QCD and searches beyond the SM. ATLAS has measured the doubledifferential cross-section as a function of rapidity and transverse jet momentum of inclusive and dijet production based on the 2010 dataset corresponding to an integrated luminosity of $37 \mathrm{pb}^{-1}$ [13]. The dijet measurement has recently been updated to the full 2011 data sample of $\mathrm{L}=4.7 \mathrm{fb}^{-1}$ [14] allowing for an improved precision w.r.t. the previous measurement. The measurements are performed in the jet rapidity range $|\mathrm{y}|<4.4(|\mathrm{y}|<2.8$ for the measurement using 2011 data), covering jet transverse 
momenta from $20 \mathrm{GeV}$ to $1.5 \mathrm{TeV}$ and dijet invariant masses from $70 \mathrm{GeV}$ to $5 \mathrm{TeV}$. Jets are identified by clustering the four-momenta of three-dimensional topological clusters built from calorimeter cells using the anti- $\mathrm{k}_{\mathrm{t}}$ algorithm with distance parameters $\mathrm{R}=0.4$ and 0.6. After correcting the energy of the jet for the impact of pile-up interactions, MC based calibration factors accounting for instrumental effects are applied as a function of the energy and pseudorapidity of the reconstructed jet [15]. The jet energy scale (JES) uncertainty is the dominant uncertainty for the inclusive jet and dijet measurements. Further corrections for detector inefficiencies and resolution are performed in an unfolding procedure based on a transfer matrix relating particle-level and reconstruction observables using the IDS method [16]. Fig. 4 shows the unfolded measured inclusive cross-sections compared to NLO pQCD calculations corrected for non-perturbative effects. Both NLOJET++ [17] and POWHEG $[18,19]$ (with the parton shower switched off) were used for the hard scatter together with various PDF sets. The data show marginally smaller cross-sections with a worse description for large jet momenta and rapidities. However, the difference between the measured cross-sections and the NLO pQCD prescriptions are in the order of the systematic uncertainty for each PDF set. Comparisons have also been performed with the POWHEG prediction, showered with either PYTHIA [12] or HERWIG [20]. Significant differences are observed between the two parton shower MCs, particularly at high $\mathrm{p}_{\mathrm{T}}$ and high rapidities.

Furthermore, measurements of the inclusive and dijet cross-section of b-jets [21] as well as the multijet cross-section [22] have been performed using ATLAS data recorded in 2010.
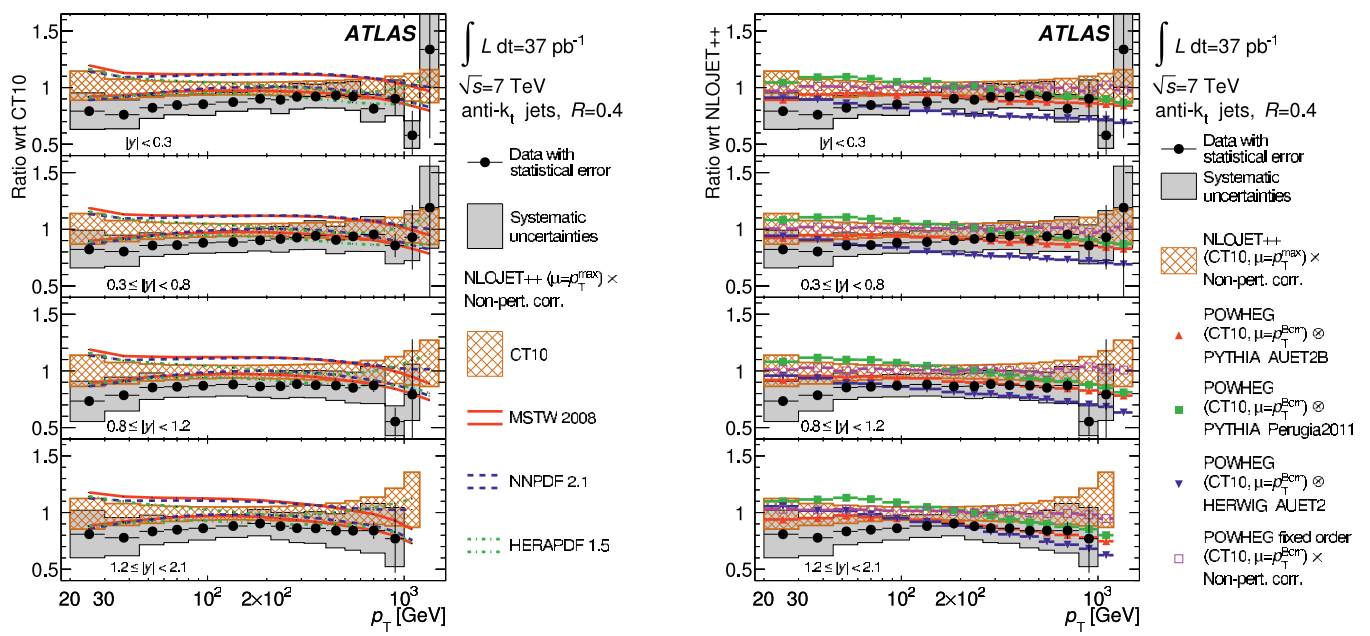

Figure 4. Ratios of the inclusive jet double-differential cross-section to the theoretical prediction obtained using NLOJET++ with the CT10 [23] PDF set in different rapidity bins for jets identified using the anti- $\mathrm{k}_{\mathrm{t}}$ algorithm with $\mathrm{R}=0.4$. The unfolded measured cross-sections are compared to theoretical prediction obtained by using NLOJET++ with different PDF sets (CT10, MSTW 2008 [24], NNPDF 2.1 [25], HERAPDF 1.5 [26]) (left) and to POWHEG predictions showered using either PYTHIA or HERWIG (right) [13].

\subsection{Jet Substructure}

The internal structure of jets is influenced by various physics processes such as hadronisation, fragmentation but also pile-up and UE (see section 2). Furthermore, decay products of heavily boosted 
particles ending up in single jets show a distinctive internal structure w.r.t. quark or gluon initiated jets. Measurements exploiting the substructure of jets therefore do not only provide insight into nonperturbative fragmentation effects [27, 28] and contributions from soft QCD background processes but can also serve as a tool to discriminate jets arising from many body decays against the dominant QCD background. Variables sensitive to the internal structure of high transverse momentum jets have recently been studied in ATLAS $[29,30]$ including $\mathrm{k}_{\mathrm{t}}$-splitting-scales, $\mathrm{N}$-subjettiness, jet width and eccentricity.

Furthermore, different algorithms allowing to remove contaminations from soft QCD processes to a given jet by making use of its characteristic internal structure are currently being investigated [31]. These so-called grooming techniques are based on the clustering ordering of the $\mathrm{k}_{\mathrm{t}}[32,33]$ and Cambridge-Aachen (C/A) [34, 35] algorithms which typically reconstruct the QCD shower in the early recombination steps while proto-jets representing the products of a heavy particle decay tend to be combined in the ultimate steps. The prunning algorithm makes use of spatial and kinematic variables characterising the branching at each point in the jet reconstruction to identify and remove soft and wide-angle radiation constituents. Similarly, the trimming procedure uses the $\mathrm{k}_{\mathrm{t}}$-algorithm to cluster the constituents of a given jet into subjets of size $R_{\text {sub }}$ whereas relatively soft subjets with $\mathrm{p}_{\mathrm{T}, \mathrm{i}} / \mathrm{p}_{\mathrm{T}}<\mathrm{f}_{\mathrm{cut}}$ are removed. Fig. 5 shows the normalised jet cross-section as a function of invariant jet mass for $\mathrm{C} / \mathrm{A} \mathrm{R}=1.2$ jets before and after applying the so-called mass-drop filtering [30] procedure. This algorithm inverts the clustering of C/A jets to identify pairs of proto-jets describing a hard and symmetric splitting. In the subsequent filtering step the constituents of this pair of jets are reclustered into various subjets using the C/A algorithm with smaller distance parameter R. To remove contributions from UE and pile-up effects only constituents within the 3 leading subjets in $\mathrm{p}_{\mathrm{T}}$ are considered to form the final jet. All of the grooming algorithms under study have been demonstrated to be powerful tools to improve the mass resolution of jets originating from heavy particle decays as well as to reduce their sensitivity to MPI processes.
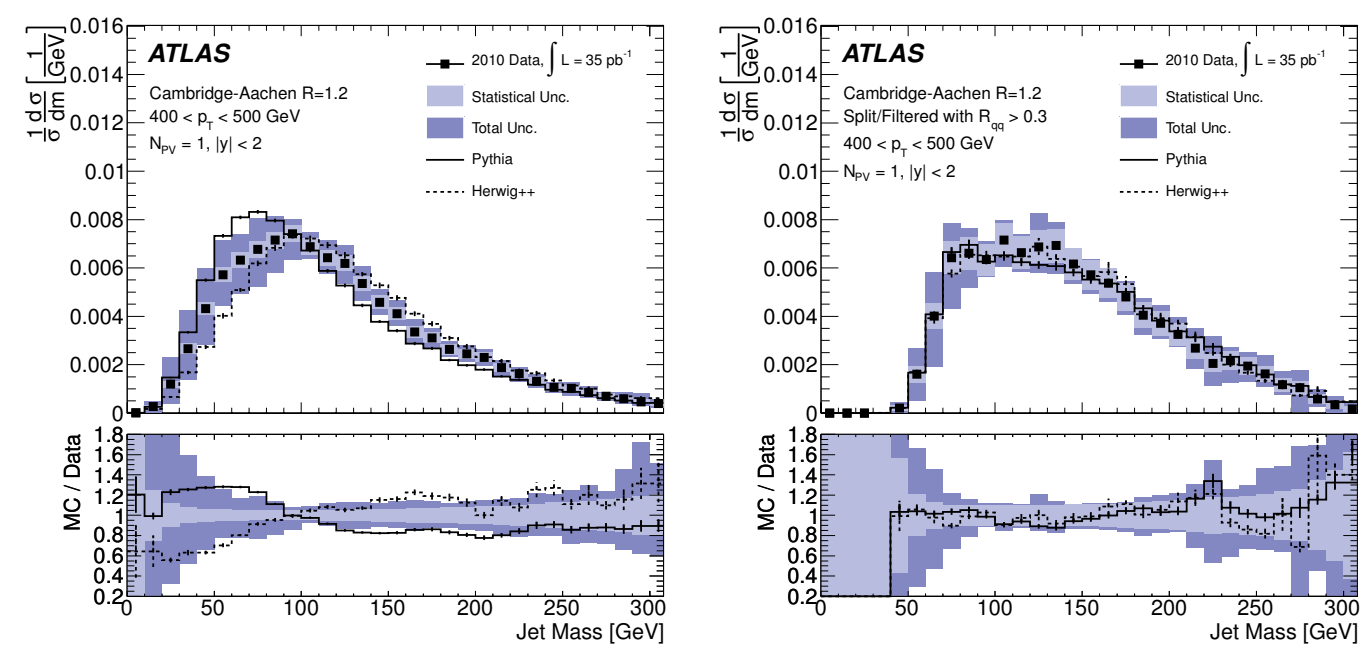

Figure 5. Normalised cross-section as a function of mass of Cambridge-Aachen jets with $\mathrm{R}=1.2$ before (left) and after (right) applying a splitting and filtering algorithm based on the jet substructure characteristics [30]. 


\section{Conclusions}

ATLAS has carried out a wide range of measurements giving insight into soft and hard QCD physics at the new energy scale accessible at the LHC. Minimum bias and underlying event studies using ATLAS data provide an important tool for additional tuning of soft QCD parameters in MC generators resulting in an improved data-MC agreement w.r.t. pre-LHC models. Studies involving high- $\mathrm{p}_{\mathrm{T}}$ jets such as the inclusive and dijet cross-section measurements have been performed in a new kinematic regime allowing to test $\mathrm{pQCD}$ in phase space regions which had not been covered by previous studies. Together, these measurements contribute to a profound understanding of the SM. Due to this, signatures deviating from SM predictions and indicating new physics processes can be detected.

\section{Acknowledgements}

The author acknowledges the financial support of the work by the DFG research training group 1054 .

\section{References}

[1] ATLAS Collaboration, JINST 3 (2008) S08003.

[2] ATLAS Collaboration, New J. Phys. 13 (2011) 053033.

[3] ATLAS Collaboration, JHEP 1207 (2012) 19.

[4] ATLAS Collaboration, ATL-PHYS-PUB-2011-009, https://cdsweb.cern.ch/record/1363300.

[5] ATLAS Collaboration, arXiv:1203.0419 [hep-ex] (2012).

[6] ATLAS Collaboration, JHEP 1205 (2012) 157.

[7] ATLAS Collaboration, Phys. Rev. D 83 (2011) 112001.

[8] ATLAS Collaboration, Eur. Phys. J. C 71 (2011) 1636.

[9] M. Cacciari, G. P. Salam and G. Soyez, JHEP 0804 (2008) 063.

[10] Rick Field, arXiv:1010.3558v1 [hep-ex] (2010).

[11] ATLAS Collaboration, arXiv:1208.0563 (2012).

[12] T. Sjostrand, S. Mrenna and P. Z. Skands, JHEP 0605 (2006) 026.

[13] ATLAS Collaboration, Phys. Rev. D 86 (2012) 014022.

[14] ATLAS Collaboration, arXiv:1112.6297 [hep-ex] (2012), accepted for publication in Phys. Rev. D.

[15] ATLAS Collaboration, arXiv:1112.6426 [hep-ex] (2011).

[16] B. Malaescu, arXiv:0907.3791 [physics.data-an] (2009).

[17] Z. Nagy, Phys. Rev. D 68 (2003) 094002.

[18] S. Alioli, P. Nason, C. Oleari and E. Re, JHEP 1101 (2011) 095.

[19] P. Nason, arXiv:0709.2085 [hep-ph] (2007).

[20] G. Corcella et al., JHEP 01, (2001) 010 (2001).

[21] ATLAS Collaboration, Eur. Phys. J. C 71 (2011) 1846.

[22] ATLAS Collaboration, Eur. Phys. J. C 71 (2011) 1763.

[23] H. -L. Lai et al., Phys. Rev. D 82 (2012) 074024.

[24] A. D. Martin, W. J. Stirling, R. S. Thorne and G. Watt, Eur. Phys. J. C 63 (2009) 189.

[25] R. D. Ball et al., Nucl. Phys. B 838 (2012) 136.

[26] H1 and ZEUS Collaborations, H1prelim-10-142, ZEUS-prel-10-018.

[27] ATLAS Collaboration, Phys. Rev. D 83 (2011) 052003. 
[28] ATLAS Collaboration, Eur. Phys. J. C 71 (2011) 1795.

[29] ATLAS Collaboration, arXiv:1206.5369 [hep-ex] (2012).

[30] ATLAS Collaboration, JHEP 1205 (2012) 128.

[31] ATLAS Collaboration, ATLAS-CONF-2012-065, https://cdsweb.cern.ch/record/1459530.

[32] S. D. Ellis and D. E. Soper, Phys. Rev. D 48 (1993) 3160.

[33] S. Catani, Y. L. Dokshitzer, M. Seymour, and B. Webber, Nucl. Phys. B 406 (1993) 187.

[34] Y. L. Dokshitzer, G. Leder, S. Moretti, and B. Webber, 1125 JHEP 9708 (1997) 001.

[35] M. Wobisch and T. Wengler, arXiv:hep-ph/9907280 [hep-ph] (1993). 